\title{
Light-emitting diodes with $31 \%$ external quantum efficiency by outcoupling of lateral waveguide modes
}

\author{
R. Windisch ${ }^{\mathrm{a})}$ and P. Heremans \\ IMEC, Kapeldreef 75, B-3001 Leuven, Belgium
}

A. Knobloch, P. Kiesel, and G. H. Döhler

Institut für Technische Physik I, Universität Erlangen-Nürnberg, D-91058 Erlangen, Germany

B. Dutta and G. Borghs

IMEC, Kapeldreef 75, B-3001 Leuven, Belgium

(Received 1 February 1999; accepted for publication 24 February 1999)

\begin{abstract}
The external quantum efficiency of light-emitting diodes (LEDs) is usually limited by total internal reflection at the semiconductor-air interface. This problem can be overcome by a combination of light scattering at a textured top surface and reflection on a backside mirror. With this design, we achieve $22 \%$ external quantum efficiency. One of the main loss mechanisms in such nonresonant cavity (NRC) light-emitting diodes is coupling into an internal waveguide. Texturing the surface of this waveguide allows the partial extraction of the confined light. In this way, we demonstrate an increase in the external quantum efficiency of NRC-LEDs to 31\%. () 1999 American Institute of Physics. [S0003-6951(99)03916-9]
\end{abstract}

Light-emitting diodes (LEDs) have a wide range of possible applications ranging from illumination purposes to optical communication systems. The efficiency of LEDs has important consequences on such applications. Due to the large difference in the refractive index, the angle of total internal reflection at the semiconductor-air interface is only $16^{\circ}$. For conventional surface-emitting LEDs, even with internal efficiencies close to $100 \%$, the external quantum efficiency is thus limited to values around $2 \%$. A promising approach to overcome this problem was proposed in $1973,{ }^{1}$ and consists of roughening one LED surface and applying a back mirror. Light, which is internally reflected at the top surface, is scattered at the rough surface and thus changes its angle of propagation. After reflection at the back mirror, it gets a second chance to escape from the semiconductor material. A technological method to realize such a nonresonant cavity (NRC) is to make use of epitaxial lift-off and consecutive Van der Waals bonding onto a mirror in order to obtain a thin-film LED. This approach was first demonstrated by Schnitzer et al. in $1993 .^{2}$

Another important issue in the design of LEDs is to avoid the generation of light underneath the top contact. In order to achieve this independently of the current level, we have implemented a current aperture fabricated by wet thermal oxidation of AlGaAs in our LEDs. Using this design, we could obtain record external efficiencies for low device currents. $^{3}$ The maximum external quantum efficiency obtained on such structures is around $20 \%$, which is still clearly below the expected internal quantum efficiency. This is due to various kinds of loss mechanisms. One of the optical loss mechanisms is absorption, either within the LED structure itself, or in the back mirror. Another reason is the formation of lateral waveguides within the active and cladding layers of the device. In this letter, we describe the enhancement of the

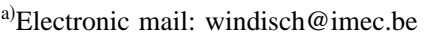

external quantum efficiency by the extraction of light from these waveguides.

The sample was grown by molecular beam epitaxy (MBE) on a GaAs substrate. The layer structure consists of an AlAs sacrificial layer for epitaxial lift-off, followed by an $800 \mathrm{~nm}$ thick $n$-doped $\mathrm{Al}_{0.25} \mathrm{Ga}_{0.75}$ As layer, a $120 \mathrm{~nm}$ thick active GaAs layer, a $130 \mathrm{~nm}$ thick and weakly $p$-doped $\mathrm{Al}_{0.3} \mathrm{Ga}_{0.7} \mathrm{As}$ spacer layer, a $130 \mathrm{~nm}$ thick $p$-doped $\mathrm{Al}_{0.98} \mathrm{Ga}_{0.02} \mathrm{As}$ layer for oxidation, and a $1 \mu \mathrm{m}$ thick $p$-doped $\mathrm{Al}_{0.25} \mathrm{Ga}_{0.75} \mathrm{As}$ top layer. In the first processing step a 60 $\mu \mathrm{m}$ diam device mesa is defined by wet chemical etching stopping in the spacer layer. Then, the $\mathrm{Al}_{0.98} \mathrm{Ga}_{0.02} \mathrm{As}$ layer is oxidized in wet $\mathrm{N}_{2}$ at temperatures around $380^{\circ} \mathrm{C}$ for 10 min, resulting in a current aperture with a diameter between 10 and $30 \mu \mathrm{m}$ depending on the oxidation temperature. A second wet chemical etching step down to the $n$-doped AlGaAs layer defines $60 \mu \mathrm{m}$ diam holes for the $\mathrm{AuGe} / \mathrm{Ni} / \mathrm{Au}$ $n$ contact, which are placed at a distance of $60 \mu \mathrm{m}$ from the device mesa edge. After applying a polyimide isolation layer and lithographically opening it on the top of the device mesa, the top contact is defined such that it makes contact to the device only on top of the oxidized area and that it extends over the edge of the device mesa to form a probing pad. The polyimide is then removed from the complete surface, except under the probing pad. The LEDs processed up to this stage are used as reference devices and further referred to as "conventional LEDs." For all other device types, a back mirror is applied by epitaxial lift-off and consecutive Van der Waals bonding onto a polyimide-coated Au mirror evaporated onto a Si wafer. The resulting flat devices with a back mirror are again characterized as a reference. For the final NRC-LEDs, surface texturing is performed in addition to the application of the mirror. For surface texturing, a densely packed monolayer of randomly distributed $400 \mathrm{~nm}$ polystyrene spheres is formed on a water surface and transferred onto the sample. ${ }^{4}$ In order to introduce a spacing in between the spheres, their 


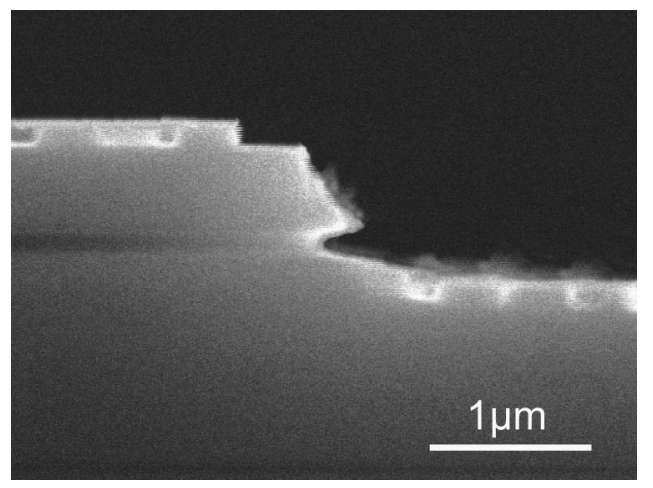

FIG. 1. Scanning electron micrograph (SEM) photograph of the textured surface on and around the mesa after removal of the spheres. The oxidized $\mathrm{AlGaAs}$ layer is clearly visible.

size is reduced to approximately $300 \mathrm{~nm}$ in oxygen plasma. This layer is used as a mask for chemically assisted ion-beam etching to an etch depth of $170 \mathrm{~nm}$. Finally, the spheres are removed from the sample. Figure 1 shows the surface texturing across and around the device mesa. In contrast, a second set of NRC-LEDs is only textured on top of the device mesa, with the surface around the device being protected by photoresist during the process of surface texturing. Figure 2 shows the resulting four types of LEDs under operation.

The output power of the LEDs is measured in a calibrated $^{5}$ setup. Figure 3(a) shows the measured light output power $P_{\text {opt }}$ as a function of the device current $I$ for the four different types of devices. The external quantum efficiency is shown in Fig. 3(b). It is defined as

$$
\eta=\frac{P_{\mathrm{opt}} / E_{\mathrm{phot}}}{I / q},
$$

with $E_{\text {phot }}=1.42 \mathrm{eV}$ and $q$ being the elementary charge. The conventional LEDs reach the expected external efficiency of slightly less than $2 \%$. The application of a back mirror without surface texturing improves the light output by a factor of 5. An analysis of the local intensity distribution shown in Figs. 2(a) and 2(b) reveals that the light output within the

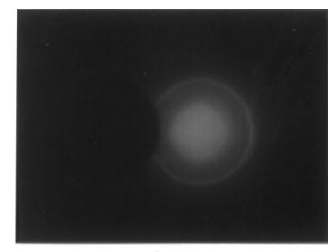

(a)

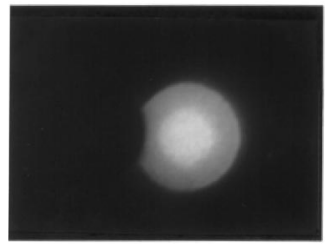

(c)

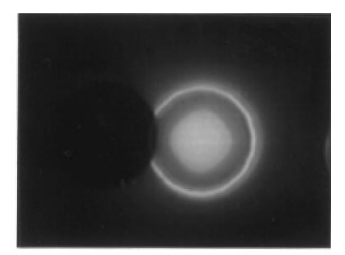

(b)

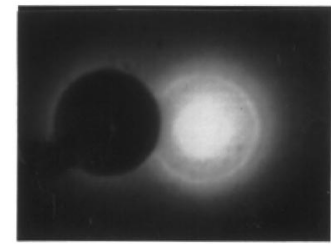

(d)
FIG. 2. CCD images of the four types of LEDs: (a) planar device without back mirror ("conventional LEDs"), (b) planar device with back mirror, (c) NRC-LED textured on top of the mesa with back mirror, and (d) NRC-LED textured everywhere with back mirror. The device currents were $45 \mu \mathrm{A}$ for the flat devices (a) and (b) and $22 \mu \mathrm{A}$ for the textured devices (c) and (d),

respectively.
Downloaded 27 Sep 2004 to 146.103.254.11. Redistribution subject to AlP license or copyright, see http://apl.aip.org/apl/copyright.jsp
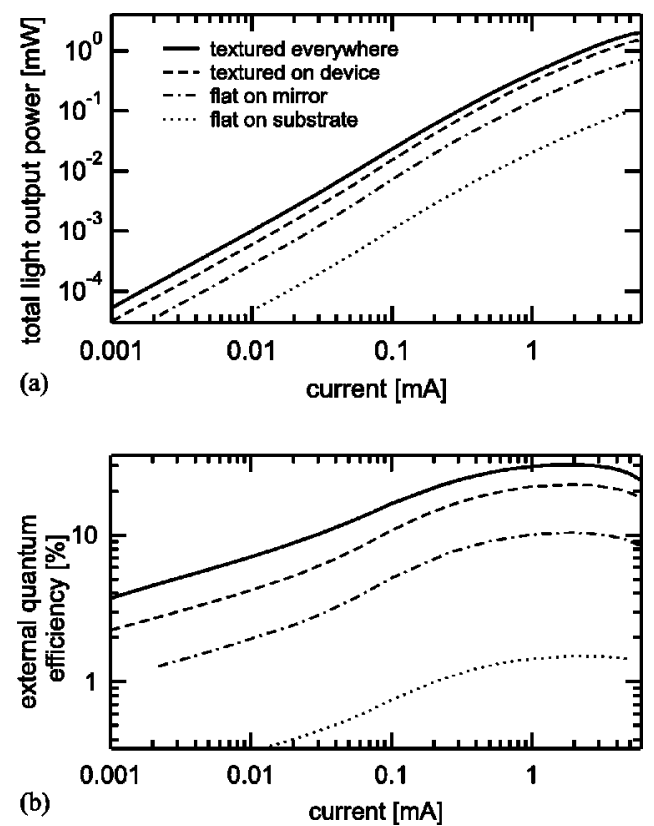

FIG. 3. Performance of the four types of LEDs: (a) $\log -\log$ plot of the total light output vs injection current and (b) external quantum efficiency vs injection current.

area of the current aperture is approximately doubled, as expected from the presence of a back mirror. The remainder of the increase in the light output is due to light coupled out at the mesa edge, which is visible as a bright ring in Fig. 2(b). This is caused by the light propagating in the waveguide formed between the top surface and the oxidized AlGaAs layer. This light can be coupled out after consecutive reflections at the $55^{\circ}$ mesa edge and the back mirror. When the top of the mesa is textured, the light is coupled out efficiently within the area of the device mesa. Such a NRC-LED, which is shown in Fig. 2(c), reaches an external quantum efficiency of $22 \%$. By texturing not only the mesa top, but also the surface around the device, the efficiency increases to as much as $31 \%$, as shown in Fig. 3(b). Figure 2(d) shows that this is due to the outcoupling of light from the surface around the device, which, in contrast to the LED only textured on top of the mesa [Fig. 2(c)], appears bright. Typical photon trajectories are drawn schematically in Fig. 4(c).

In order to quantify the fraction of light emitted around the device, the intensity distribution has been measured with a charge-coupled device (CCD) camera. The result is shown in Fig. 4(a). Due to the limited dynamic range of the CCD camera, the curves of Fig. 4(a) are obtained by superimposing two sets of data. The intensity distribution on top of the device mesa is measured directly. For the area around the device mesa, the same measurement is performed with a higher drive current. The symbols in Fig. 4(a) represent the intensity averaged over $10 \times 10 \mu \mathrm{m}^{2}$ spots measured at varying radial distances $r$ from the device center. It is scaled to the appropriate total light output, assuming a currentindependent intensity distribution. The decay of the emission in the area around the device mesa can be fitted by a function proportional to $(1 / r) \times \exp \left(-r / r_{0}\right)$. The extension of the curves in Fig. 4(a) beyond the device edge, i.e., for $r$ $>30 \mu \mathrm{m}$, represents this fit for each of the devices. The light output for the NRC-LED textured only on top of the device AlP license or copyright, see http://apl.aip.org/apl/copyright.jsp 

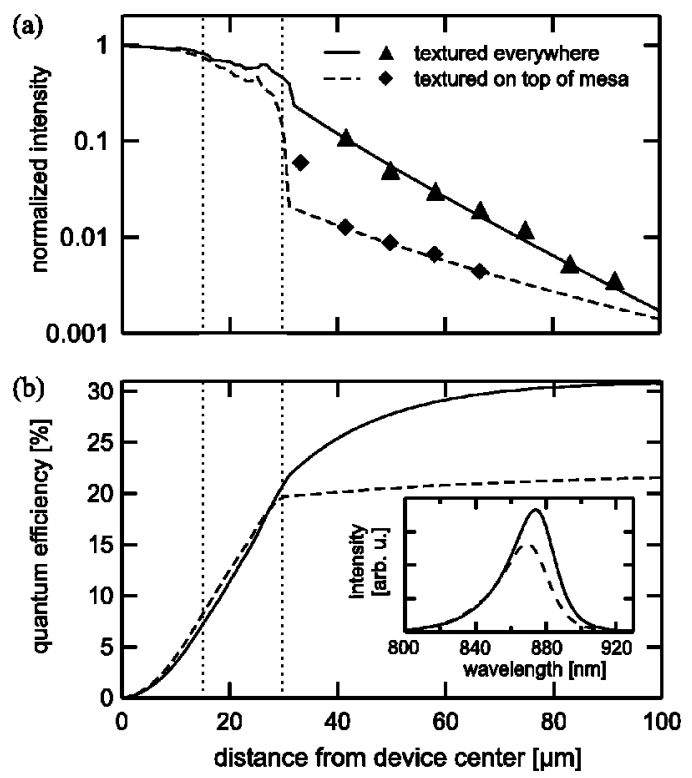

(c)

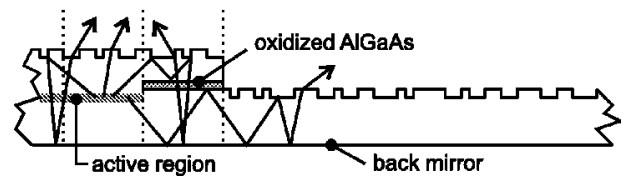

FIG. 4. (a) Intensity distribution of the two types of NRC-LEDs with mirror. (b) Integrated areal intensity as a function of the radius. The inset shows the spectrum of both device types. (c) Schematic drawing of the completely textured sample including typical photon trajectories. The scale of this drawing is identical to the axis scale in (a) and (b).

drops by more than one order of magnitude next to the mesa edge [see also Fig. 2(c)]. However, a small amount of light is also emitted from the waveguide in the flat structure around the device mesa. This is probably due to scattering at the unintended roughness introduced by wet chemical etching. If the LED is textured everywhere, a much larger amount of light is coupled out in the area surrounding the mesa. In addition to the enhancement in the external efficiency, this also leads to a faster radial decay of the emission. The decay length obtained from the fit in Fig. 4(a) for this device is $r_{0}=18 \mu \mathrm{m}$, in contrast to $r_{0}=45 \mu \mathrm{m}$ for the LED only textured on top of the mesa.
The intensity emitted within a circular area of radius $r$ can be obtained by the integration of the radial intensity distribution after multiplication by $r$. The result normalized to the external quantum efficiency of the respective device is shown in Fig. 4(b). It shows that within the area of the device mesa, the amount of extracted light is almost identical for both types of NRC-LEDs. As expected, the improvement of the external efficiency from $22 \%$ to $31 \%$ originates from the light emitted around the device mesa. This has a consequence for the emission spectrum. The light in the waveguide has a longer path length within the active GaAs layer. Thus, it is expected that the part of the light emitted at photon energies above the GaAs band-gap $E_{g}$ is mostly reabsorbed. Recycling of the absorbed photons is unlikely, since in the area around the current aperture the carrier density is small, and thus, the radiative recombination efficiency is low. Hence, only photons with an energy below $E_{g}$ can be extracted efficiently outside the device mesa. This results in an effective shift of the emission spectrum of these LEDs to longer wavelengths, as shown in the inset of Fig. 4(b).

We have presented highly efficient nonresonant cavity LEDs with a maximum external quantum efficiency of $31 \%$ obtained by the outcoupling of guided modes. We conclude that the light from guided modes can be efficiently extracted from planar waveguides by the application of a random surface texturing. This technique should also be applicable for other types of LEDs like microcavity LEDs.

The authors thank W. Van de Graaf for the MBE growth of the samples and S. Peeters for help in processing. This work was in part supported by the EC under Contract No. 22641 (OIIC). One of the authors (R.W.) acknowledges the EC for his Marie Curie Fellowship No. ERBFMICT972046.

${ }^{1}$ A. Bergh and H. Saul, U.S. Patent No. 3,739,217 (1973).

${ }^{2}$ I. Schnitzer, E. Yablonovitch, C. Caneau, T. J. Gmitter, and A. Scherer, Appl. Phys. Lett. 63, 2174 (1993).

${ }^{3}$ R. Windisch, P. Heremans, B. Dutta, M. Kuijk, S. Schoberth, P. Kiesel, G. H. Döhler, and G. Borghs, Electron. Lett. 34, 1153 (1998).

${ }^{4}$ H. W. Deckman and J. H. Dunsmuir, Appl. Phys. Lett. 41, 377 (1982).

${ }^{5}$ R. Windisch, P. Heremans, B. Dutta, S. Nemeth, A. Knobloch, G. H. Döhler, and G. Borghs, Proc. SPIE (in press). 